\title{
Effect of Goji berry consumption on physiological, biochemical and traditional Chinese medicine (TCM) outcomes
}

\author{
G.M.R. Gonçalves ${ }^{1}$, L. Zhao ${ }^{2}$, S. Patel ${ }^{1}$ and B.A. Graf ${ }^{1}$ \\ ${ }^{1}$ Food and Nutrition, Department of Health Professions, Faculty of Health, Psychology and Social Care, \\ Manchester Metropolitan University, M15 6BG Manchester, UK and ${ }^{2}$ Health Academy, OL10 1AA Heywood, UK.
}

Traditional Chinese medicine (TCM) prescribes food as medicine ${ }^{(1)}$, and Goji berry (Lycium barbarum) were used to treat imbalances of 'Yin', 'Yang' and 'Qi' for $>2500$ years ${ }^{(2)}$. Recent animal studies report that Goji berry reduced total cholesterol, body weight and fasting blood glucose $\mathrm{s}^{(3,4,5,6)}$. In human trials Goji berry were evaluated as part of a complex juice, which contained a large number of other ingredients and reported effects could not be attributed to Goji berry ${ }^{(7,8)}$. Thus, we investigated the effect of dried Goji berry in a double-blind randomised controlled study design. Stratified by BMI, participants were randomly allocated into Goji berry ( $20 \mathrm{~g} / \mathrm{d}$, $\mathrm{n}=14$ ) or control group $(15.7 \mathrm{~g}$ green raisins $/ \mathrm{d}, \mathrm{n}=13)$. On day 1 and day 21 of the study, conventional outcomes (line $1-8$, Table 1$)$ and TCM outcomes (line 9-14, Table 1) were evaluated.

Table 1. Effect of Goji berry consumption on physiological, biochemical and traditional Chinese medicine (TCM) outcomes.

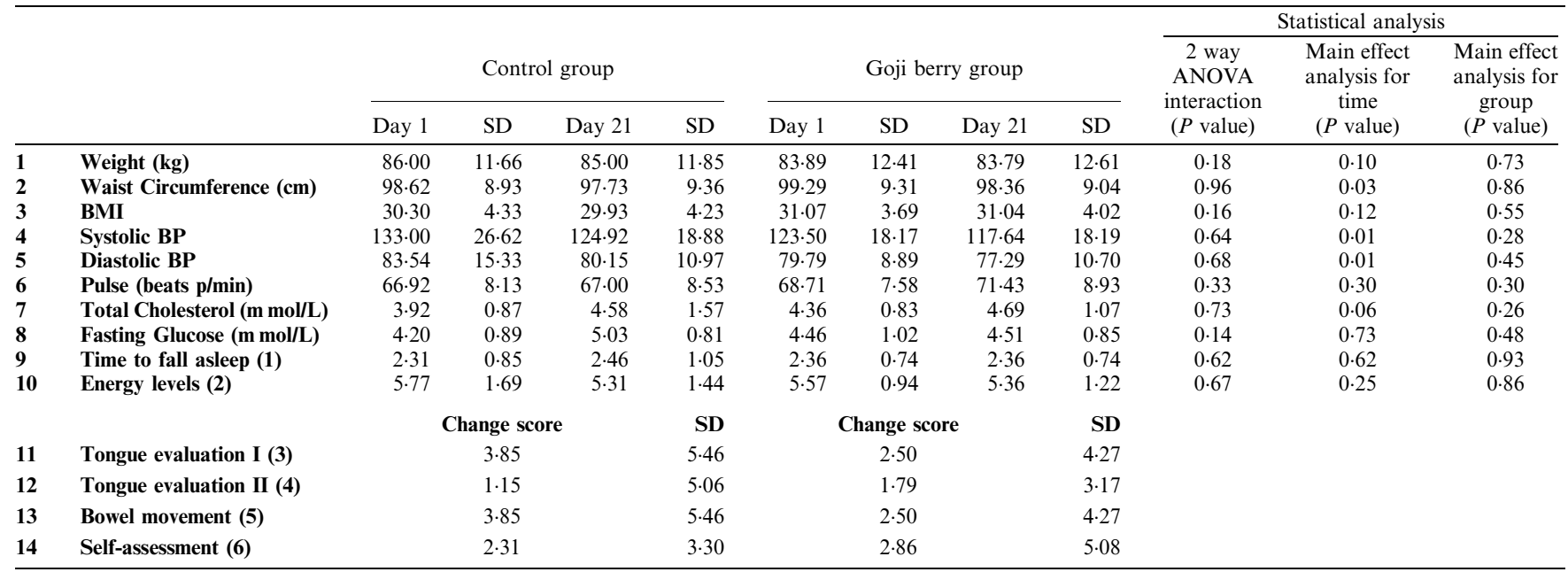

(1) Time to fall asleep was categorised: $1=>20 \mathrm{~min}, 2=11-20 \mathrm{~min}, 3=5-10 \mathrm{~min}$; (2) Energy levels: $1=$ Extreme low, $5=$ Medium, $10=$ Excellent, (3) Tongue evaluation I: TCM tongue diagnosis: $-10=$ Reduction of health $(2$ or more tongue areas changed negatively), $-5=$ Slight reduction $(1$ area changed negatively), $0=$ No change, $5=$ Slight improvement $(1$ area changed positively), $10=$ Improvement $(2$ or more areas changed positively; (4) Tongue evaluation II: TCM tongue diagnosis based on tongue photograph by external TCM practitioner: same scale as before; (5) Bowel movement and (6) Self-assessment: $-10=$ Negative change, $-5=$ Slight negative change, $0=$ No change, $5=$ Slight positive change, $10=$ Positive change .

Two-way ANOVA (group and time interaction analysis) revealed that there was no difference between control and Goji berry group $(P>0 \cdot 16)$. Main effect analysis showed that some outcomes changed over time (waist circumference, $P=0 \cdot 03$; systolic BP $P=0.01$; diastolic BP, $P=0 \cdot 01$ ). These changes however occurred in both, control and Goji berry group. Therefore changes seen were either a) due to a factor not measured in the study or b) Goji berry AND green raisins delivered the same bioactivity. In summary, this study cannot confirm that Goji berry consumption has beneficial effects.

1. Maciocia G (2005) The Foundations of Chinese Medicine. 2nd ed., New York, USA: Elsevier Health Sciences.

2. Potterat $\mathrm{O}$ (2010) Journal of medicinal plant and natural product research 76, 7-19.

3. Ming M, Guanhua L, Zhanhai Y et al. (2009) Journal Food Chemistry 113(4), 872-877.

4. Luo Q, Cai Y, Yan J et al. (2004) Life Sciences 76(2), 137-149.

5. Jing L, Cui G, Feng Q \& Xiao Y (2009) African Journal of traditional, complementary alternative medicines 6(4), 579-584.

6. Li XM (2007) International Journal of Biological Macromolecules 40(5), 461-465.

7. Amagase H \& Nance DM (2011) Journal of the American College of Nutrition 30(5), 304-309.

8. Amagase H \& Nance DM (2012) Journal of Food Research 1(2), 3-12. 